\title{
TARGETING THE TUMOR METABOLISM BY OXAMATE POTENTIATES THE IMPACT OF CHEMOTHERAPEUTICS IN COLORECTAL CANCER CELLS
}

\author{
Gizem Calibasi-Kocal ${ }^{1}$, Cagri Cakici ${ }^{2}$, Feriha Toksoz ${ }^{1}$, Seniz Inanc-Surer ${ }^{3}$, \\ Tolga Sever $^{4}$, Yasemin Basbinar ${ }^{1}$, Turkan Yigitbasi ${ }^{2}$
}

${ }^{1}$ Department of Translational Oncology, Institute of Oncology, Dokuz Eylul University, Izmir- Turkey.
${ }^{2}$ Department of Biochemistry, Faculty of Medicine, Istanbul Medipol University, Istanbul- Turkey.
${ }^{3}$ Department of Biochemistry, Faculty of Medicine, Dokuz Eylul University, Izmir- Turkey.
${ }^{4}$ Department of Translational Oncology, Institute of Health Sciences, Dokuz Eylul University, Izmir- Turkey.

Address for Correspondence: Gizem Calibasi-Kocal., E-mail: gizem.calibasi@deu.edu.tr

Received: 31.07.2021; Accepted: 06.09.2021; Available Online Date: 20.09.2021

(C) Copyright 2021 by Dokuz Eylül University, Institute of Health Sciences - Available online at https://dergipark.org.tr/en/pub/jbachs

Cite this article as: Calibasi-Kocal G, Cakici C, Toksoz F, Inanc-Surer S, Sever T, Basbinar Y, Yigitbasi T. Targeting the tumor metabolism by oxamate potentiates the impact of chemotherapeutics in colorectal cancer cells. J Basic Clin Health Sci 2021; 3: 205- 212.

\begin{abstract}
Purpose: Cancer cells promote lactate formation via pyruvate rather than oxidative phosphorylation by programming their metabolism to maintain proliferation under the Warburg effect. It has shown that the altered metabolic phenotype with activation of lactate dehydrogenase-A in the cancer cell may affect survival, chemotherapy resistance, and metastasis. In this direction, studies are focusing on reprogramming cancer metabolism and increase the effectiveness of chemotherapy. In this study, the main aim was to target the Warburg phenotype via the inhibition of lactate dehydrogenase with the combination of sodium oxamate and current colorectal cancer treatment options such as 5-fluorouracil and irinotecan.
\end{abstract}

Methods: The effect of chemotherapeutics on the cellular behavior was evaluated by real-time cytotoxicity and migration analysis systems, and metabolic phenotype was assessed by measuring lactate, lactate dehydrogenase expression, and reactive oxygen species levels.

Results: According to the results, the viability and migration of colorectal cancer cells were significantly decreased with the combination of chemotherapeutics and sodium oxamate which decreases lactate levels.

Conclusion: As a result, the combination of sodium oxamate with chemotherapeutics hinders the cancer cell viability and migration by changing metabolic phenotype with decreased lactate.

Keywords: Colorectal cancer, tumor metabolism, lactate, sodium oxamate, Warburg effect

\section{INTRODUCTION}

Combinations of drugs, which include the use of conventional cytotoxic drugs and targeted drugs that prevent abnormal cellular signaling in cancer, are trying to increase the success of the treatment by reducing the risk of clonal selection of tumor cells. In combination therapy regimens, targeted therapies provide specificity to inhibit or alter the functions of 
molecular targets such as growth factors, cell surface receptors, or enzymes associated with tumorigenesis and cancer progression $(1,2)$. At this point, researchers and clinicians are in search of new treatment options that can be used in the management of colorectal cancer. Altered metabolic factors and pathways of cancer cells have been suggested as important targets for the development of new and combination drugs to improve response to conventional chemotherapy. Although significant progress has been made in understanding the genetic and epigenetic factors that promote tumor development and metastasis, the progress in the development of new cancer drugs has been slow due to changes in tumor metabolism which induce tumor progression and metastatic phenotype (3).

Metabolic reprogramming of cancer cells is a wellknown feature of malignancy which is about aberrant glucose metabolism $(4,5)$. Cancer cells produce lactate by the lactate dehydrogenase enzyme via pyruvate, instead of the conversion 'glucosepyruvate-acetyl CoA' by oxidative phosphorylation based metabolic reprogramming to support their proliferation and anabolic growth under the conditions known as Warburg's effect $(6,5,7)$. At this point, researches for the modification of cancer cell-specific metabolism and maximizing the efficiency of chemotherapy are increasing. Therefore, this study was based on the role of tumor metabolism on chemotherapy efficacy. The main aim of this study is to maximize the effects of current treatment options of 5-Fluorouracil (FU) and irinotecan (IRI) on colorectal cancer cells by using sodium oxamate (OX) as a lactate dehydrogenase inhibitor.

\section{MATERIALS AND METHODS}

\section{Cell culture and chemicals}

Human colorectal adenocarcinoma cell line HCT116 (ATCC $\AA$ CCL-247 ${ }^{\mathrm{TM}}$ ) and HT-29 (ATCC $\otimes$ HTB-38 ${ }^{\mathrm{TM}}$ ) were purchased from ATCC (American Type Culture Collection, Manassas, VA, USA). All cells were maintained in McCoy's 5A medium (Gibco, Life Technologies, NY, USA) supplemented with $10 \%$ fetal bovine serum (FBS) (Gibco, Life Technologies, NY, USA) and 1\% penicillin/streptomycin (Gibco, Life Technologies, NY, USA) in a humidified incubator at $37^{\circ} \mathrm{C}$ with $5 \%$ CO2. Sodium oxamate, 5 -Fluorouracil (5FU), and irinotecan were purchased from Sigma Aldrich. Drugs were administered after 24 hours of cell seeding.

\section{Determination of drug doses}

The IC50 doses of the drugs to be used on the selected colorectal cancer cell lines were determined primarily for drug administration to the experimental groups. The IC50 dose (half maximal inhibitory concentration) is the drug concentration that makes the $50 \%$ reduction on reduces cell viability from the start of the experiment and represents the net loss of cells. For this, changes in cell index and number because of serial dilution of chemotherapeutics and sodium oxamate in different doses applied to HCT116 and HT-29 cells were evaluated using the Real-time Cell Analysis System (xCelligence Real-Time Cell Analyzer, ACEA, Biosciences, San Diego, CA) and IC50 doses were calculated. The inhibition rate was calculated in percent using the time zero at the beginning of the experiment (time zero, $(\mathrm{Tz})$ ), control (C), and measurement ( $\mathrm{Ti})$ values at the concentrations of the compounds. For HCT116: 5Fluorouracil $17 \mu \mathrm{M}$; Irinotecan $100 \mu \mathrm{M}$; Sodium oxamate $25 \mathrm{mM}$. For HT-29: 5-Fluorouracil $120 \mu \mathrm{M}$; Irinotecan $21 \mu \mathrm{M}$; Sodium oxamate was determined as $25 \mathrm{mM}$. To examine the effects of fluorouracil, irinotecan, and sodium oxamate on CRC cells, experimental groups will be formed in which chemicals are applied alone and sodium oxamate in combination. After the determination of IC50 doses, experimental groups were determined, and experimental applications were carried out.

\section{Real-time cell proliferation experiment}

Real-time cell proliferation experiments were performed by using XCELLigence RTCA SP device (ACEA Biosciences, Inc., San Diego, CA, USA) which was placed in a humidified incubator at $37{ }^{\circ} \mathrm{C}$ in $5 \%$ $\mathrm{CO} 2$. In this system, analysis are based on the impedance changes caused by the cell attachment, spreading, and proliferation to the bottom of the well where the gold electrodes are covered. Cells were seeded into the wells of $96 \mathrm{E}$-plates which were specifically designed for the platform, with $1 \times 10^{4}$ cells per well (in total $200 \mu \mathrm{L}$ of cell culture medium). The cells were visualized as real-time for 24 hours at $37^{\circ} \mathrm{C}$ in an environment containing $5 \% \mathrm{CO} 2$, and at the end of the 24th hour, all the culture medium in the $96 \mathrm{E}$ plate was withdrawn and different concentrations of drugs were applied. Cell index measurement of cells was taken every 15 minutes for 48 hours. Three replications of each drug and combinations, as well as the control group, were used in the cell 
proliferation experiment, and each experiment was repeated three times.

\section{Real-time cell migration experiment}

Real-time cell migration experiments were performed by using $x$ CELLigence RTCA DP device (ACEA Biosciences, Inc, San Diego, CA, USA) which was placed in a humidified incubator at $37^{\circ} \mathrm{C}$ in $5 \% \mathrm{CO} 2$. In this system, analysis was conducted with the CIMPlate consisting of an upper and a lower chamber separated by a filter membrane with an $8 \mu \mathrm{M}$ pore diameter to the assessment of migration. The $8 \mu \mathrm{m}$ porous membrane allows cell migration through the filter membrane of the upper chamber where the microelectrodes were embedded. When cells migrate, a signal was generated because of the altered electric impedance. Cells were seeded into the upper chamber at 40,000 cells/well in the presence of a serum-free cell culture medium and with the specific chemotherapeutic application. The culture medium containing serum was applied to the bottom chamber for migration. Due to the migratory characteristics of cells, cell index values representing the passage through the pores were obtained by measuring the changes in impedance values every 15 minutes for 48 hours. The migratory property of the cell was presented by expressing the migration of untreated control cells as $100 \%$. Three replications of each drug and combinations, as well as the control group, were used in the cell proliferation experiment, and each experiment was repeated three times.

\section{L-Lactate assay}

using the L-Lactate Assay Kit (Colorimetric) (Abcam, ab65331) based on the principle of reading the intensity of the color at $450 \mathrm{~nm}$ because of the interaction of the formed product, which is released because of the oxidation of lactate with lactate dehydrogenase. The amount of lactate will be normalized compared to the control group. Analysis of lactate formation was carried out after the 48th hour of chemotherapeutic applications to have information about the conversion of pyruvate to lactate. The lactate concentration was measured by

\section{Statistics}

Statistical analysis was performed using GraphPad Prism 5.0 (GraphPad Software, Inc., La Jolla, CA, USA). The non-parametric Mann Whitney $U$ test was used to evaluate the differences within the evaluated parameters. Numerical values of text and figures are expressed as mean \pm standard error. P-value is accepted as $\leq 0.05$. All experiments were repeated in three times.

\section{RESULTS}

Colorectal cancer cells, HCT116 and HT-29 were cultured with IC50 doses (half maximal inhibitory concentration) for 5-Fluorouracil, irinotecan, and sodium oxamate. To be sure that sodium oxamate reduces the amount of lactate, lactate analysis was performed primarily in the groups that were administered different drugs and combinations. When the lactate concentration of the control group in HCT116 cells was compared with the other groups (FU, IRI, OX, FU-OX, IRI-OX, FU-IRI-OX, and FUIRI), a significant decrease was observed in all groups compared to the control group ( $p \leq 0.05)$. The same significant reduction was observed in HT-29 cells $(p \leq 0.05)$ (Figure 1).
A

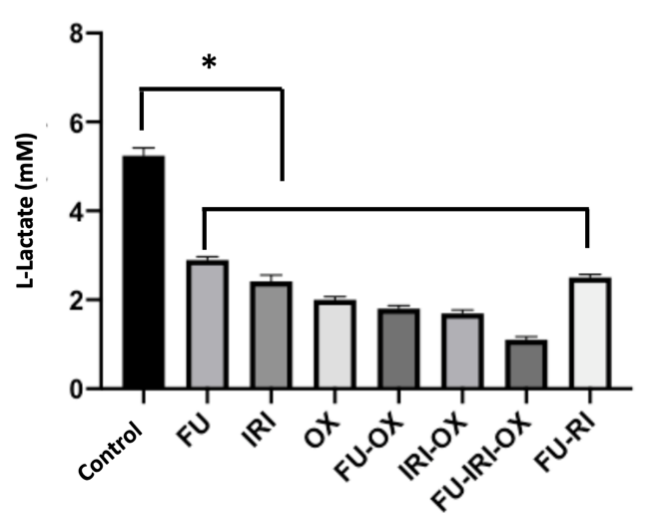

B

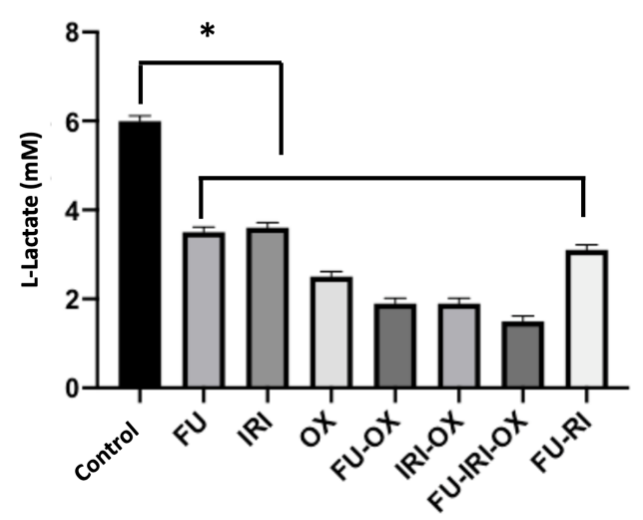

Figure 1. Lactate plots in HCT116 and HT-29 cells. (FU: 5-Fluorourasil, IRI: Irinotecan, OX: Sodium oxamate) 

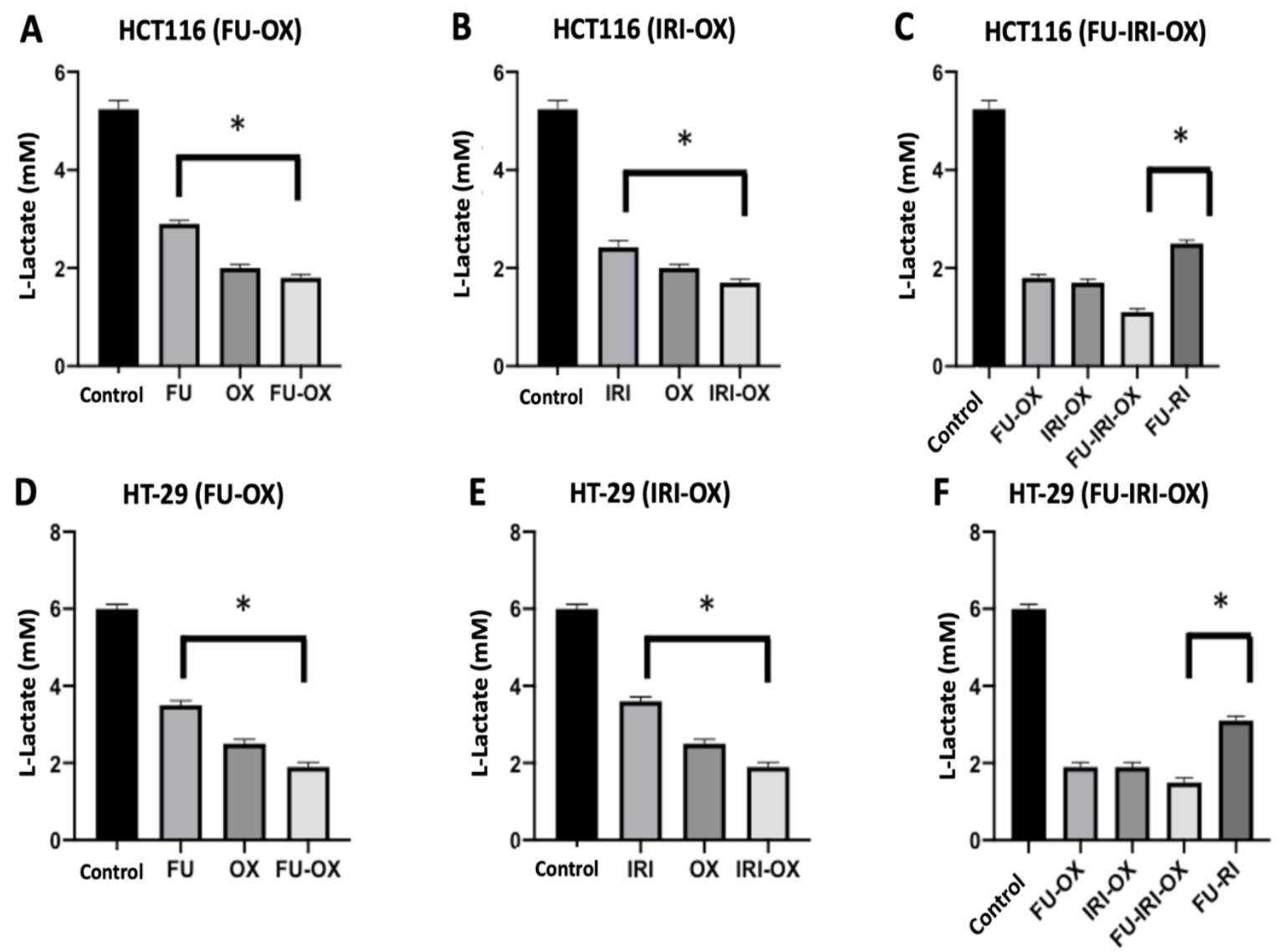

Figure 2. Evaluation of the Lactate plots according to different drug combinations in HCT116 and HT-29 cells. ${ }^{*} p \leq 0.05$ was considered significant. (FU: 5-Fluorouracil, IRI: Irinotecan, OX: Sodium oxamate)

When the effect of the application of 5-fluorouracil and irinotecan alone or together with sodium oxamate on HCT116 cells on lactate concentration was examined, it was observed that the amount of lactate in the medium was significantly reduced because of the co-administration of sodium oxamate with drugs $(p \leq 0.05)$ (Figure 2). The same result was observed for HT29 cells (Figure 2).

According to the viability results, 5 fluorouracil/sodium oxamate combinations have decreased the viability more than only 5-fluorouracil applied colorectal cancer cells for both HCT116 and HT-29 cells ( $p \leq$ 0.05 ). Irinotecan/sodium oxamate combination was decreased the viability more than only irinotecan applied colorectal cancer cells for both
HCT116 and HT-29 cells ( $p \leq 0.05)$. 5fluorouracil/sodium oxamate combination has also decreased the viability more than only 5 -fluorouracil applied colorectal cancer cells for both HCT116 and HT-29 cells ( $p \leq 0.05)$ (Figure 3 ).

When the migration results were evaluated, there is no significant reduction for 5-fluorouracil in combination with sodium oxamate, and 5fluorouracil/irinotecan in combination with sodium oxamate for both HCT116 cells. FU-OX and IRI-OX combinations have inhibited the migration more than only chemotherapeutic applied HT-29 cells. The same significant reduction was also seen on 5fluorouracil/irinotecan and its co-administration with sodium oxamate on HT-29 cells $(p \leq 0.05)$ (Figure 4). 

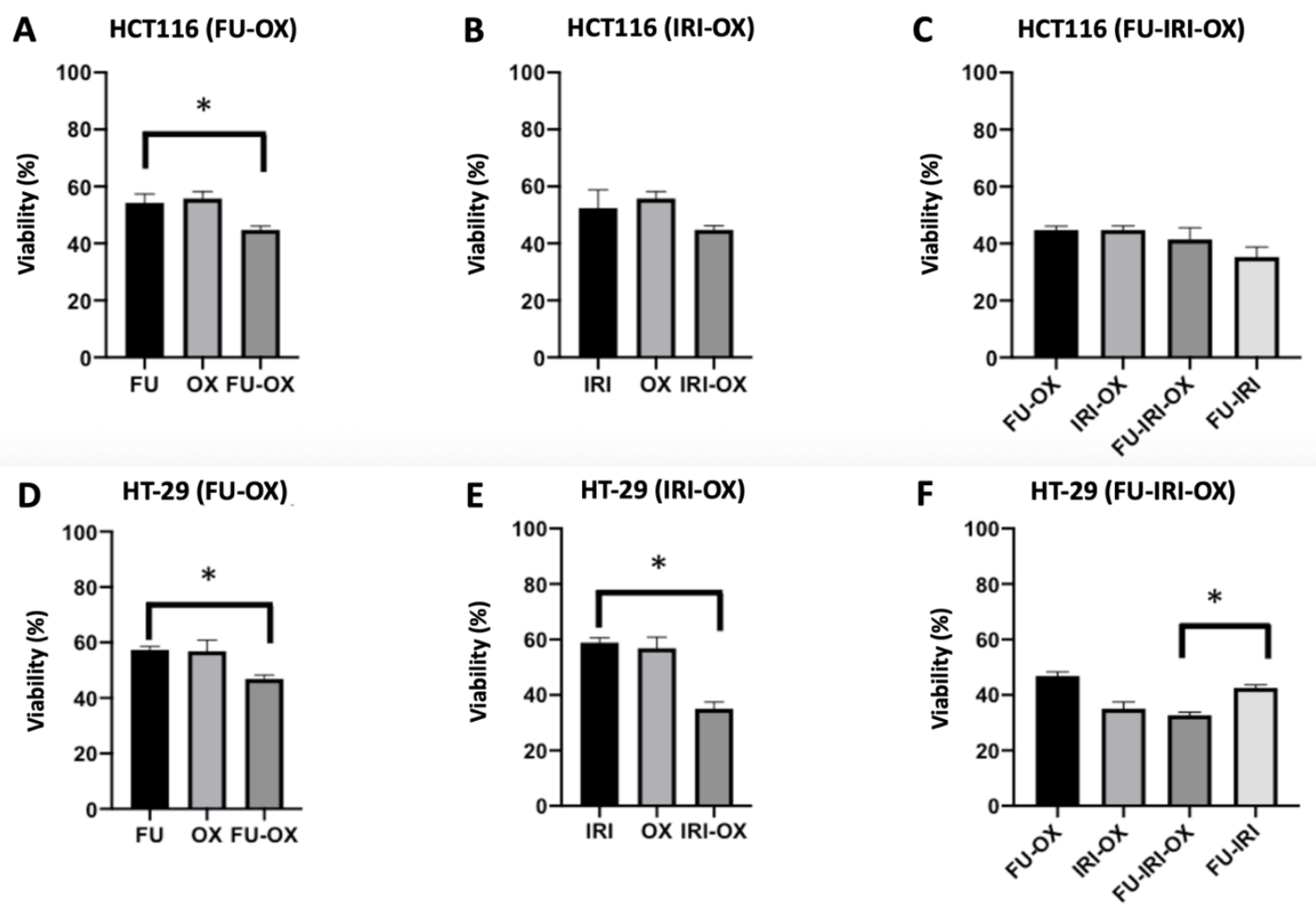

Figure 3. Viability plots of HCT116 and HT-29 cells. A) The effect of administration of 5-fluorouracil alone and in combination with sodium oxamate, B) irinotecan alone and in combination with sodium oxamate, C) 5-fluorouracil/irinotecan and its coadministration with sodium oxamate on the viability of HCT116 cells. D) The effect of administration of 5-fluorouracil alone and in combination with sodium oxamate, E) irinotecan alone and in combination with sodium oxamate, F) 5fluorouracil/irinotecan and its co-administration with sodium oxamate on the viability of $\mathrm{HT}-29$ cells. ${ }^{*} \mathrm{p} \leq 0.05$ was considered significant. (FU: 5-Fluorouracil, IRI: Irinotecan, OX: Sodium oxamate)

A

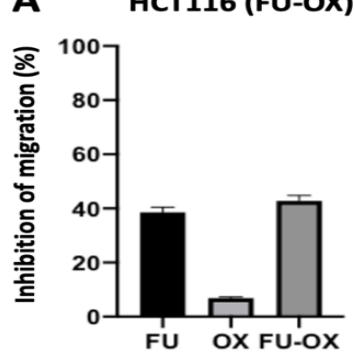

D

HT-29 (FU-OX)

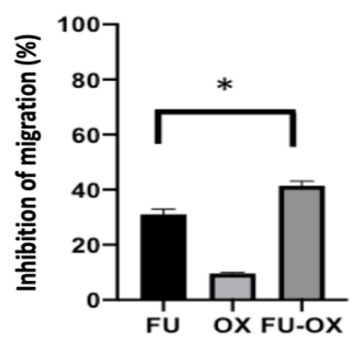

B

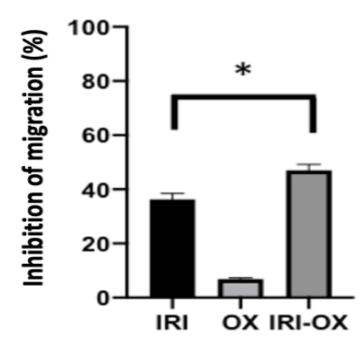

E

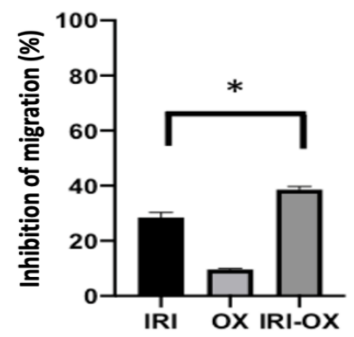

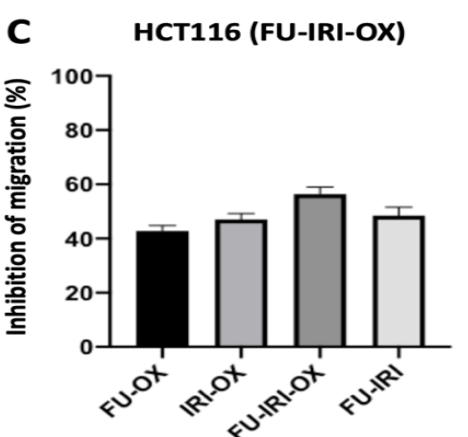

$\mathbf{F}$ HT-29 (FU-IRI-OX)

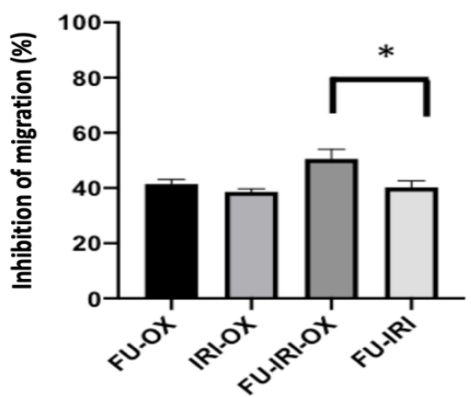

Figure 4. Migration plots of HCT116 and HT-29 cells. A) The effect of administration of 5 -fluorouracil alone and in combination with sodium oxamate, B) irinotecan alone and in combination with sodium oxamate, C) 5 -fluorouracil/irinotecan and its co-administration with sodium oxamate on the viability of HCT116 cells. D) The effect of administration of 5-fluorouracil alone and in combination with sodium oxamate, E) irinotecan alone and in combination with sodium oxamate, F) 5fluorouracil/irinotecan and its co-administration with sodium oxamate on the viability of $\mathrm{HT}-29$ cells. ${ }^{*} \mathrm{p} \leq 0.05$ was considered significant. (FU: 5-Fluorouracil, IRI: Irinotecan, OX: Sodium oxamate) 


\section{DISCUSSION}

\section{Effect of sodium oxamate on lactate levels}

When the effect of administration of sodium oxamate in combination with 5-fluorouracil and irinotecan to HCT116 cells on the production of lactate was examined, the combination of 5-fluorouracil/sodium oxamate compared to the use of 5-fluorouracil alone; irinotecan/sodium oxamate combination compared to irinotecan alone; and 5-fluorouracil/irinotecan/sodium oxamate combination significantly reduced lactate production compared to 5-fluorouracil/irinotecan combination ( $p \leq 0.05$ ) (Figure 2). When the lactate graphs of HT-29 cells are examined, the combination of 5-fluorouracil/sodium oxamate compared to the use of 5-fluorouracil alone; irinotecan/sodium oxamate combination compared to irinotecan alone; and 5-fluorouracil/irinotecan/sodium oxamate combination significantly reduced lactate production compared to 5-fluorouracil/irinotecan combination ( $\mathrm{p} \leq$ 0.05) (Figure 2).

In the literature, a decrease/inhibition of LDH-A activity with sodium oxamate application and, consequently, a decrease in lactate production and a decrease in lactate concentrations have been shown. In the study of Zhao et al., in which they used sodium oxamate among $20-120 \mathrm{mM}$ dose range to inhibit LDH-A in gastric cancer cells, a dose-dependent decrease in lactate concentration was detected with sodium oxamate administration (8). In the study of Seliger et al. in which they modulated lactate with sodium oxamate application on glioma cells, they found a dose-dependent decrease in LDHA activity and lactate concentration with sodium oxamate administration (9). Liu et al. reported that the administration of sodium oxamate in the dose range of 20-100 mM on gastric carcinoma cell lines was dose-dependently significant in lactate production found a reduction (10). In the study of Zhai et al. on nasopharyngeal cells and Yang et al. on lung cancer cells, found a dose-dependent decrease in LDHA activity with sodium oxamate administration $(11,12)$.

\section{Effect of sodium oxamate combination of} chemotherapeutics on CRC cell viability

When the viability effect of sodium oxamate application in combination with 5-fluorouracil and irinotecan to HCT116 cells was examined, it was determined that 5 -fluorouracil/sodium oxamate combination significantly reduced cell viability compared to the application of 5-fluorouracil alone $(p \leq 0.05)$. In addition, the combination of irinotecan/sodium oxamate compared to irinotecan alone, and the combination of 5 fluorouacil/irinotecan/sodium oxamate did not significantly reduce cell viability compared to the fluorourocil/irinotecan combination (Figure 3). When the viability graphs of HT-29 cells are examined, the combination of 5 -fluorouracil/sodium oxamate is compared to the use of 5-fluorouacil alone; irinotecan/sodium oxamate combination compared to irinotecan alone; and the use of 5fluorouacil/irinotecan/sodium oxamate combination significantly reduced cell viability compared to 5fluorouacil/irinotecan combination ( $p \leq 0.05$ )(Figure 3). Studies conducted by various groups have shown that LDH inhibition influences increased sensitivity to chemotherapy administered to cancer cells $(13,14)$. For example, Zhou et al. reported that the combination of sodium oxamate with taxol significantly reduced viability and induced apoptosis in taxol-resistant breast cancer cells $(13,14)$. However, there is no study in the literature evaluating the use of chemotherapeutics currently used in CRC with sodium oxamate.

HCT116 and HT-29 are genetically different cell lines, while HCT116 harbors the KRAS G13D mutation; HT29 has BRAF V600E mutation. Differences observed because of administration of the same drugs and doses $s$ can be interpreted through genetic difference. There are publications in the literature regarding the metabolic phenotypes and gene expression patterns of CRCs that differ in terms of KRAS and BRAF mutations $(15,16,17)$. In our study, the synergistic inhibitory effect of sodium oxamate in combination with existing chemotherapeutics on the viability of CRC cells is clearly seen. In line with its effect, it can be interpreted that the aggressive phenotype acquired by the cancer cell is prevented by the inhibition of the LDH enzyme and the decrease in lactate levels.

\section{Effect of sodium oxamate on CRC cell migration}

When the migration inhibitory effect of sodium oxamate application in combination with 5fluorouracil and irinotecan to HCT116 cells was examined, it was determined that irinotecan/sodium oxamate combination significantly reduced migration compared to irinotecan alone $(p \leq 0.05)$. However, the combination of 5 -fluorouracil/sodium oxamate compared to the use of 5-fluorouracil alone; and 5fluorouracil/irinotecan/sodium oxamate combination compared to the 5-fluorouracil/irinotecan combination 
did not significantly reduce migration (Figure 4). When the migration graphs of HT-29 cells are examined, the combination of 5-fluorouracil/sodium oxamate compared to the use of 5-fluorouracil alone; irinotecan/sodium oxamate combination compared to irinotecan alone; the combination of 5fluorouracil/irinotecan/sodium oxamate significantly reduced migration compared to the combination of 5fluorouracil/irinotecan ( $p \leq 0.05)$ (Figure 4).

Liu et al. reported that the use of sodium oxamate in the $20-100 \mathrm{mM}$ dose range reduced the invasive character of gastric carcinoma cells, although they have not conducted their studies on the use of sodium oxamate in combination with existing chemotherapeutics (10). In the literature, there are studies in which LDH-A was inhibited by using siRNA (not by chemical use). In these studies, it was reported that the invasive feature of cancer cells decreased with the inhibition of LDH-A. For example, Arseneault and colleagues showed that cell migration was significantly reduced because of inhibition of LDHA with siRNA in breast cancer cells (18). In the study conducted by Jiang et al. on bladder cancer, it was shown that inhibition of LDH-A with siRNA significantly reduced the expression of epithelialmesenchymal transition-related proteins (E-cadherin) and the invasive/migratory properties of bladder cancer cells (19).

\section{CONCLUSION}

Consequently, when sodium oxamate is used in combination with 5- fluorouracil and irinotecan, the viability and migration of the colorectal cancer cells significantly decreased compared to 5-fluorouracil and irinotecan alone. Reducing lactate by sing sodium oxamate as a lactate dehydrogenase inhibitor supports the decrease in viability and migration. Numerous studies of drugs that modulate cancer metabolism are being conducted. Therefore, it is likely that additional drugs for cancer metabolism for cancer therapy could be developed alone or in combination with conventional therapy, targeted therapy and immunotherapy.

Conflict of Interest: The authors declare no conflict of interest. Funding: This project was supported by Dokuz Eylul UniversityScientific Research Projects, 2018.KB.SAG.072.

Peer-review: Externally peer-reviewed.

\section{REFERENCES}

1. Persidis A. Cancer multidrug resistance. Nat Biotechnol 1999; 17(1); 94-5.

2. Crawford $\mathrm{S}$. Is it time for a new paradigm for systemic cancer treatment? Lessons from a century of cancer chemotherapy. Front Pharmacol. 2013; 4: 68.

3. Galluzzi L, Kepp O, Vander Heiden MG, et al. Metabolic targets for cancer therapy. Nat Rev Drug Discov 2013; 12(11): 829-46.

4. Schwartz L, Supuran CT, Alfarouk KO. The Warburg Effect and the Hallmarks of Cancer. Anticancer Agents Med Chem 2017; 17(2); 16470.

5. Vander Heiden MG, Cantley LC, Thompson CB. Understanding the Warburg effect: the metabolic requirements of cell proliferation. Science 2009; 324(5930): 1029-33.

6. Doherty JR, Cleveland JL. Targeting lactate metabolism for cancer therapeutics. J Clin Invest 2013; 123(9): 3685-92.

7. Ward PS, Thompson CB. Metabolic reprogramming: a cancer hallmark even warburg did not anticipate. Cancer Cell 2012; 21(3): 297308.

8. Zhao Z, Han F, Yang S, et al. Oxamate-mediated inhibition of lactate dehydrogenase induces protective autophagy in gastric cancer cells: involvement of the Akt-mTOR signaling pathway. Cancer Lett 2015; 358(1): 17-26.

9. Seliger C, Leukel P, Moeckel S, et al. Lactatemodulated induction of THBS-1 activates transforming growth factor (TGF)-beta2 and migration of glioma cells in vitro. PLoS One 2013; 8(11): e78935.

10. Liu X, Yang Z, Chen Z, et al. Effects of the suppression of lactate dehydrogenase $A$ on the growth and invasion of human gastric cancer cells. Oncol Rep 2015; 33(1): 157-62.

11. Zhai $X$, Yang $Y$, Wan J, et al. Inhibition of LDH-A by oxamate induces $\mathrm{G} 2 / \mathrm{M}$ arrest, apoptosis and increases radiosensitivity in nasopharyngeal carcinoma cells. Oncol Rep 2013; 30(6): 298391.

12. Yang $Y$, Su $D$, Zhao $L$, et al. Different effects of LDH-A inhibition by oxamate in non-small cell lung cancer cells. Oncotarget 2014; 5(23): 1188696.

13. Fiume L, Manerba $M$, Vettraino $M$, et al. Impairment of aerobic glycolysis by inhibitors of lactic dehydrogenase hinders the growth of 
human hepatocellular carcinoma cell lines.

Pharmacology 2010; 86(3): 157-62.

14. Zhou M, Zhao $Y$, Ding $Y$, et al. Warburg effect in chemosensitivity: targeting lactate dehydrogenase-A re-sensitizes taxol-resistant cancer cells to taxol. Mol Cancer 2010; 9: 33.

15. Hutton JE, Wang $X$, Zimmerman $L J$, et al. Oncogenic KRAS and BRAF Drive Metabolic Reprogramming in Colorectal Cancer. Mol Cell Proteomics 2016; 15(9): 2924-38.

16. Lundberg IV, Wikberg ML, Ljuslinder I, Li X, Myte $\mathrm{R}$, et al. MicroRNA Expression in KRAS- and BRAF-mutated Colorectal Cancers. Anticancer Res 2018; 38(2): 677-83.

17. Sagaert X, Vanstapel A, Verbeek S. Tumor Heterogeneity in Colorectal Cancer: What Do We Know So Far? Pathobiology 2018; 85(1-2): 72-84

18. Arseneault R, Chien A, Newington JT, et al. Attenuation of LDHA expression in cancer cells leads to redox-dependent alterations in cytoskeletal structure and cell migration. Cancer Lett 2013; 338(2): 255-66.

19. Jiang $F$, Ma $S$, Xue $Y$, et al. LDH-A promotes malignant progression via activation of epithelialto-mesenchymal transition and conferring stemness in muscle-invasive bladder cancer. Biochem Biophys Res Commun 2016; 469(4): 985-92. 